\title{
Sleep does not facilitate insight in older adults
}

\author{
Ursula DEBARNOT ${ }^{1,2,3,4}$, Marta ROSSI ${ }^{5,6}$, Sophie SCHWARTZ ${ }^{1,2,3}$, Ugo FARAGUNA ${ }^{5}$, Laura \\ SEBASTIANI ${ }^{5}$
}

${ }^{1}$ Department of Neuroscience, Faculty of Medicine, University of Geneva, Geneva, Switzerland

${ }^{2}$ Geneva Neuroscience Center, University of Geneva, Geneva, Switzerland

${ }^{3}$ Swiss Center for Affective Sciences, University of Geneva, Geneva, Switzerland

${ }^{4}$ Inter-university Laboratory of Human Movement Science-EA 7424, University Claude Bernard Lyon 1, Villeurbanne, France.

${ }^{5}$ Dipartimento di Ricerca Traslazionale e delle Nuove Tecnologie in Medicina e Chirurgia, Università degli Studi di Pisa, Italy.

${ }^{6}$ School of Life Sciences, University of Sussex, Brighton, United Kingdom.

\section{Correspondence:}

Ursula DEBARNOT, Inter-university Laboratory of Human Movement Science-EA 7424, University Claude Bernard Lyon 1 Villeurbanne, France.

Email: ursula.debarnot@univ-Lyon1.fr 
Sleep has been shown to foster the process of insight generation in young adults during problem solving activities. Aging is characterized by substantial changes in sleep architecture altering memory consolidation. Whether sleep might promote the occurrence of insight in older adults as well has not yet been tested experimentally. To address this issue, we tested healthy young and old volunteers on an insight problem solving task, involving both explicit and implicit features, before and after a night of sleep or a comparable wakefulness period. Data showed that insight emerged significantly less frequently after a night of sleep in older adults compared to young. Moreover, there was no difference in the magnitude of insight occurrence following sleep and daytime -consolidation in aged participants. We further found that acquisition of implicit knowledge in the task before sleep potentiated the gain of insight in young participants, but this effect was not observed in aged participants. Overall, present findings demonstrate that a period of sleep does not significantly promote insight in problem solving in older adults. 
Sleep is beneficial for human memory performance. Both declarative and procedural memories are improved by post-learning sleep as compared to an equivalent period of wakefulness (for overviews see [declarative, Born \& Wilhelm, 2012]; [procedural, Song, 2009]). A prevalent hypothesis posits that off-line consolidation processes occurring during sleep promote the transformation of a labile memory trace into a more robust and stable one (Stickgold and Walker, 2007). A substantial body of experimental evidence suggests that patterns of brain activity elicited while practicing a memory task may spontaneously reemerge during sleep (Buhry, et al., 2011,Diekelmann and Born, 2010). Memory replay might not only allow the maintenance or strengthening of isolated memory elements, but also facilitates cross-item integration and remodeling of existing memory stores (Igloi, et al., 2015,Tamminen, et al., 2010,Walker and Stickgold, 2010). Such restructuration of knowledge may explain why sleep is also believed to help reframing and solving problems.

Until recently, the relationship between sleep and memory in older adults has sparked off little interest (Mander, et al., 2014,Van Der Werf, et al., 2009), although aging is often characterized by some memory impairment, changes in sleep architecture, as well as brain and neurochemical alterations (Harand, et al., 2012). Beside an increase of sleep fragmentation that occurs with aging, the most striking change in sleep architecture is the dramatic decrease in time spent in slow-wave sleep (SWS) accompanied by a reduction in the number and amplitude of slow oscillations (Petit, et al., 2004). Accordingly, Klerman et al. (2013) further demonstrated that sleep fragmentation in elderly may be primarily caused by the decrease capacity to maintain non rapid eye movement (NREM) sleep. By contrast, rapid eye movement (REM) sleep shows relatively less decline (Carskadon and Rechtschaffen, 2005,Scullin, 2012), with the duration of REM sleep episodes through the 
night remaining relatively constant in older adults (Van Cauter, et al., 2000). Therefore, agerelated changes in sleep architecture and especially the decrease in SWS may disrupt memory consolidation processes in elderly adults.

Age-related modifications in sleep patterns and memory functions are well documented, yet their possible interaction has rarely been investigated and existing studies yielded seemingly inconsistent results with respect to the nature of the learning. In the declarative memory domain, Backhaus et al. (2007) reported that older participants showed less improvement on a paired-associates word task after a night of sleep than young participants, with performance decrement correlating with SWS reduction in the older participants. By contrast, Wilson et al. (2012) reported an equivalent magnitude of sleepdependent memory benefit both in older and young adults. Future investigations are thus awaited to resolve the seemingly inconsistent results obtained in this research domain.

In the procedural memory domain, sleep-related consolidation of motor skills has been shown to differ depending on the explicit or implicit nature of the learning (Doyon, et al., 2009,Song, 2009). Using an explicit procedural learning task (i.e. explicit sequential finger tapping task), Wilson et al. (2012) reported reduced sleep-dependent changes in older adults, while Tucker et al. (2011) found it preserved (note that no correlation was found between overnight memory improvement and sleep architecture). Diverging results have been found for implicit procedural memory consolidation in elderly. For instance, Spencer et al. (2006) observed that performance on an implicit procedural task did not improve following a night of sleep in older adults, while Nemeth et al. (2010) found contrasting results with off-line improvement of implicit motor skill in the same type of population both over sleep and wake consolidation intervals. Altogether, these findings suggest that both 
explicit and implicit aspects in the learning tasks should be taken into account when studying the effect of sleep on procedural memory in older adults.

In the seminal study by Wagner et al. (2004), young adults were instructed to solve an insight problem called the number reduction task (i.e. NRT; modified version developed by Thurstone and Thurstone, 1941). The task implies series of eight numbers that need to be combined sequentially following specific rules to obtain a final numeric result. Critically, and unmentioned to the participants, all strings contain the same underlying structure with the last three responses mirroring the three preceding ones which allows the second response to be identical to the final one. Thus, participants can either generate responses faster through an implicit acquisition of the underlying structure, or may explicitly discover the final result that can be derived from the very early steps in the series (i.e. emergence of insight). Wagner et al. (2004) reported that time spent in sleep, but not in wake, increased the individual's capacity to express insightful behavior, hence reaching the final result faster. This study thus provides experimental support to the common belief that "sleeping on a problem" can potentiate the emergence of a solution (Robertson, 2009). In some follow-up studies using the NRT, Yordanova and colleagues further explored the respective roles of SWS and REM in the generation of insight into the task regularity, i.e. gain of explicit knowledge (Yordanova, et al., 2009,Yordanova, et al., 2008). They found that insight increased following an early-night sleep, rich in SWS, but not when the first exposure to NRT and the subsequent test were filled with a late-night REM-rich period (Yordanova, et al., 2008). Moreover, they also reported that participants who developed implicit knowledge on the NRT before early-night sleep had an increased probability of discovering the hidden rule the day after. Such findings challenge those reported by Cai et al. (2009) who demonstrated 
that compared with quiet rest and SWS sleep, REM enhances the assimilation of information into past experience, and thus fosters the formation of novel associations.

Until now, however, whether age-related changes in sleep patterns impact the reorganization of knowledge, such as a switch between implicit to explicit knowledge, and facilitate insight in problem solving tasks is still unknown. Therefore, the main aims of the current study were (i) to compare insight in the NRT after a night of sleep between young and old adults, (ii) to test whether a night of sleep rather than a similar interval of wakefulness might influence insight problem solving in old adults, and (iii) to explore whether the occurrence of insight may be differentially affected with respect to the type of knowledge - implicit vs no-knowledge - acquired either before sleep or wakefulness intervals. To this end, we studied performance on the NRT before and after a night of sleep in young (YoungSleep) and older adults (OldSleep), and after a similar wakefulness interval without sleep in older adults (OldWake). We hypothesized that the occurrence of insight in problem solving would be higher in the OldSleep group than the OldWake group. Since SWS declines with age, we expected that the YoungSleep group would gain more insight overnight as compared to the OldSleep group. Finally, we further hypothesized that participants in the sleep groups who gained implicit knowledge on problem solving before sleep would have higher probability of getting insight into the solution in the morning.

\section{MATERIALS AND METHODS}

\section{Participants}

Seventy-four participants, $20-71$ years of age, were tested. Participants were divided into 3 groups: YoungSleep (27 participants, 17 women, mean age \pm SD: $25.3 \pm 3.5$ yrs), 
OldSleep (23 participants, 10 women, mean age \pm SD: $60.5 \pm 4.6 y r s$ ) and OldWake (24 participants, 14 women, mean age \pm SD: $58.6 \pm 6$ yrs). Elderly participants were randomly assigned in the sleep or wake groups. Individuals were excluded if they had been diagnosed with a neurological disease, congestive heart failure, or a myocardial infarction, or had a history of stroke, head trauma, or heart surgery. Additionally, we excluded participants who used sleep-affecting medications, as well as extreme evening- and morning-type individuals (Horne \& Ostberg, 1976), or regular nappers. In elderly participants, additional exclusion criteria were a Mini-Mental State score lower than 27 (Folstein, et al., 1975) and a Geriatric Depression Scale (Yesavage, et al., 1982) with a depression score of more than five points. It is worth mentioning that the Mini-Mental State score provided fast assessment of episodic memory which is well known to be impacted by the effect of age (Carcaillon et al. 2009). Young participants with a score higher than 9 on the Beck Depression inventory (Beck, et al., 1961) were not included in the study. Participants in the YoungSleep and OldSleep groups were also asked to fill out the Pittsburg Sleep Quality Index to assess sleep quality and quantity (Buysse, et al., 1989). This test was administered to exclude participants who were experiencing obvious sleep disturbances. We further excluded participants who discovered the hidden rule during the first NRT session ( $12 \%$ of the initially tested participants) and stopped the inclusion when each group reached 22 participants who did not find the rule. All participants were instructed to be drug, alcohol, and caffeine free during the experiment. This study was approved by the local ethics committee at the University of Pisa, and all participants signed an informed consent form. The procedure used in this experiment was explained, and instructions regarding the NRT and questionnaires were given, although no information was provided about the objectives of the study or about the dependent 
variables of interest.

Neuropsychological and sleep assessments

A neuropsychological test battery was administered to each participant to provide an estimate of cognitive functioning including working memory and mental flexibility. The following neuropsychological tests were administered to all participants: Corsi Block Test (Milner, 1971), the 'Trail Making Test' part A and B (Reitan, 1992), and the Forward and backward Digit Span subtests of the Wechsler Adult Intelligence Scale III (Wechsler, 1981). The purpose of these neuropsychological tests was to assess differences in cognitive capacities between young and aged participants, and to ensure that both aged groups did not differ for these variables. Actigraphy was used to control and collect mean sleep duration data and sleep/wake patterns in YoungSleep and OldSleep participants. Each of the $\mathrm{n}=44$ subjects who took part in the sleep groups was equipped with a wrist actigraph immediately after the first NRT session (i.e. at around $10 \mathrm{pm}$ ) until the beginning of the second NRT session (i.e. 9am). Beside the actigraphic assessment for the sleep groups, we used questionnaires to evaluate the subjective quality and quantity of sleep during the night following the first NRT training. Importantly and as OldWake participants did not wear actigraph, they were explicitly asked to not nap between the first and second NRT sessions. Subjective measures of alertness and fatigue were also collected in all participants using the Stanford Sleepiness Score before the first and the second NRT sessions (Hoddes, et al., 1972).

\section{Number Reduction Task (NRT)}

We used the same version of the NRT as developed in the study by Wagner et al. (2004; see Figure 1). Each participant was shown different strings of eight digits on a 
computer screen. Each string was composed by the digits ' 1 ', ' 4 ', and ' 9 '. For each string, participants had to determine the digit defined as the 'final result' of the string, by sequentially processing the digits pairwise from left to right according to two simple rules. The 'identity rule' states that the result of two identical digits is that same digit (for example, ' 9 ' and ' 9 ' results in ' 9 ', as in response 1 in Fig. 1 ), while the 'difference rule' states that the result of two non-identical digits is the remaining third digit of this three-digit system (for example, ' 9 ' and ' 1 ' results in ' 4 ' as in response 2 in Fig. 1). After processing the first two digits, the rules are applied on the preceding result and the next digit. Instructions stated that only the final result was to be determined for each trial and this could be done at any time by pressing the space bar after any entered digit.

\footnotetext{
*** Insert Figure 1 here $* * *$
}

Importantly, all strings had the same underlying structure: response sequences always had an $A B A C C A B$ structure (with $A, B$, and $C$ corresponding to one of the digits 1,4 , or 9), i.e., the last three responses mirroring the preceding three responses. This structure also implies that the second response in each trial was identical to the final one. Thus, participants may generate responses faster through an implicit acquisition of the underlying structure, and show actual insight by pressing the 'space bar' key already after the second response. Because actual digit strings and responses differed from trial to trial, discovering the hidden rule may not arise from repeating the same finger movements.

The D, G, and J keys on the PC keyboard (AZERTY) were labeled 1, 4, and 9, and served as response keys. Each response appeared on the screen and remained there until 
the end of the trial, thereby forming a response string below the stimulus string. Each response was limited to $4 \mathrm{~s}$. For each series, participants would thus generate a string of seven responses, with the last one indicating the final result to be confirmed by pressing the 'space bar' on the keyboard. In case of a correct final result, all digit responses on the screen changed their color to green during $2 \mathrm{~s}$, whereas they changed to red in case of an incorrect final result. The next stimulus string would then appear $0.5 \mathrm{~s}$ after the colored feedback.

Reaction times (RTs) were recorded for each key press from the first response (R1), which was the time from string appearance to the first key press, to the $7^{\text {th }}$ response, which was the final result of the string. The RTs of the other responses $(R 2, R 3, R 4, R 5, R 6, R 7$, space bar) were measured as the time between the previous and the current key press. Each sequence with a false final response was discarded from RT analysis.

\section{Experimental procedure}

Participants were tested individually in a sound-attenuated room. The young and older sleep groups were subjected to the first NRT session at 9pm and performed the second NRT session on the next morning at 9am. The OldWake group was tested on the first NRT session at 9am and was re-tested on the same day at 9pm. The first NRT session consisted of 3 blocks including 30 trials each, while the second NRT session comprised 10 blocks of 30 trials each. Previous studies using this version of NRT have shown that the 'solvers' gain explicit knowledge that emerges within 10 experimental blocks (Haider and Rose, 2007,Yordanova, et al., 2009). The first NRT session was preceded by extensive standardized instructions given by the experimenter, and included a short practice block of five trials. To ensure correct understanding of the 'identity' and 'difference' rules, the practice block was repeated as long as the participant did not perform five trials without mistakes. To exclude 
that insight into the hidden rule had taken place spontaneously between the first and the second NRT practice, the experimenter asked the participants to explain how they proceeded during the task and whether they had to report something regarding the task at the end of the first NRT session. Similar questions were requested at the end of the second NRT session in order to probe whether participants acquired explicit knowledge related to the hidden rule or not.

\section{Statistical analysis}

The dependent variables were the number of participants who gained spontaneously explicit knowledge (ExK) of the hidden rule during the post-interval session and the response times. The former was assessed using mean RT and questionnaire data in order to confirm the shortcut in sequential responding. Chi-square $2 \times 2$ tables were used to determine whether young rather than older participants might gain more insight after a night of sleep (YoungSleep vs OldSleep), and to test whether sleep had a beneficial impact on the emergence of insight for the older adults groups (OldSleep vs OldWake). Then, using these RTs, we applied a similar method as used in Yordonova et al. (2008) to identify the knowledge states for all participants regarding the hidden regularity before and after the sleep or daytime interval. For each block and participant, we computed the mean RTs of the R3 and R4 (i.e. unpredictable responses), and the mean RTs of R6 and R7 (i.e. predictable responses), in each single string. Then, we performed a one-way analysis of variance (ANOVA) with repeated-measures with Predictability as within-subjects factor (R3/R4 vs. R6/R7). This was done in the second and the third block of the first NRT session. Participants were assigned either to the pre-implicit knowledge (prelmk) subgroup if the $p<.01$ criterion was met in at least one of the two tested blocks, otherwise to the pre-no knowledge 
(preNoK) subgroup. The same analysis was performed in each block of the 10 blocks of the second NRT session, for each participant except those who gained ExK of the hidden rule. Based on the results from the one-way ANOVA, participants were assigned to the postimplicit knowledge (post-Imk) subgroup if the $p<.01$ criterion was met in at least three blocks of the ten tested blocks, otherwise to the post-no knowledge (post-NoK) subgroup Whenever appropriate, Bonferroni post-hoc comparisons were performed. Then, to confirm that ImK, but not NoK participants, were significantly faster for R6/R7 predictable responses compared with unpredictable responses R3/R4, we performed two repeated measures ANOVAs, for the first and second NRT sessions, with Group and Knowledge (preNoK vs prelmK, and postNoK vs postlmK) as between-subjects factor and Predictability (unpredictable R3/R4 vs predictable R6/R7) as within-subjects factor. Finally to evaluate whether the level of knowledge acquired before the consolidation interval (preNoK or prelmK) had an impact on the transformation in ExK, we performed Fisher exact tests between subgroups. Group scores on neuropsychological test as well as actigraphy data were compared using Student unpaired $t$ tests between groups. We used Statistica workpackage (Statsoft Inc., Tulsa, OK, USA) for data analysis. Throughout, the results will be given as mean \pm standard error of the mean, and a $p<.05$ was considered as statistically significant.

\section{RESULTS}

Questionnaires and sleep assessment

An overview of the neuropsychological data for the 3 groups is shown in Table 1. There was no significant difference among groups for any variable. Likewise, there was no 
difference on 7-point scale $(1$ = being most alert) of the Stanford Sleepiness Scale between sessions or between groups: mean values for YoungSleep group were $1.68(.10)$ before the first NRT session and 1.45 (.11) before the second session and the corresponding scores in the OldSleep groups were $1.45(.11)$ and $1.27(.10)$, and $1.27(.10)$ and $1.55(.11)$ in the OldWake group. Global Pittsburg Sleep Quality scores were $4.36(.40)$ in the YoungSleep group, 4.45 (.57) in the OldSleep group, and $3.86(.39)$ in the OldWake group. No significant difference was found between the 3 groups, thus attesting that all participants had a good quality of sleep. Analysis of actigraphy data combined with subjective assessment of the night of sleep revealed that the OldSleep group slept earlier and got up earlier than the YoungSleep group (Table 2). No significant differences in the main measures of sleep based on the actigraphy were observed between age groups (including sleep latency, total sleep time, total sleep period, and sleep efficiency). No other significant differences in sleep measures were found between age groups.

\section{*** Insert Table 1 and 2 here ***}

\section{Behavioural performance}

We tested whether young adults might gain more insight after a night of sleep as compared to older adults, since the former should have more SWS than the latter. Chisquare tests were calculated to investigate differences between insight solvers of the YoungSleep group ( $n=11 ; 50 \%)$ and those of the OldSleep group ( $n=4 ; 18 \%)$. The results revealed that more young adults gained insight compared to older adults following a night of $\operatorname{sleep}\left(\chi^{2}(1)=4.95, p=.026\right)$. 


\section{$* * *$ Insert Figure 2 here $* * *$}

Second, we aimed to determine whether a night of sleep might facilitate the emergence of insight in older adults. The results showed that during the second NRT session, four participants in the OldSleep group (18\%) and five participants in the OldWake group (23\%) gained insight of the hidden rule $\left(\chi^{2}(1)=.14, p>.05\right)$, hence demonstrating that a night of sleep had no significant beneficial effect on problem solving in older adults compared to an equivalent interval of wakefulness.

Next, we explored whether the level of knowledge acquired before the consolidation interval (either sleep or daytime) might impact the emergence of insight. To address this question, we distinguished participants who had gained some implicit knowledge from those who displayed no such knowledge after the first NRT session based on significant difference between unpredictable and predictable responses in the mirror structure of NRT string (see the Statistical analysis section). In total, $41 \%$ of the participants developed ImK on the NRT during the first NRT session (YoungSleep group: $45.5 \%$, OldSleep group: $45.5 \%$ and OldWake group: $31.8 \%$ ), while the others did not (preNoK) (Table 3). Based on this subdivision, we then stratified the participants as a function of their knowledge after the consolidation interval, as tested during the second NRT session, into three levels of knowledge: post-NoK, post-ImK, and ExK (or "insight"; Figure 3). Two separate repeated measures ANOVAs with Group and Knowledge (preNoK vs preImK, and postNoK vs postImK) as between-subjects factor and Predictability (unpredictable R3/R4 vs predictable R6/R7) as within-subjects factor, confirmed that ImK participants generated the predictable responses 
(R6-R7) significantly faster compared to the unpredictable (R3-R4), whereas NoK subjects did not (PREDICTABILITY X KNOWLEDGE interaction: $F_{1,60}=37.20, p<.0001, \eta_{p}{ }^{2}=.38$ for the first NRT session and $\mathrm{F}_{1,40}=40.04, p<.0001, \eta_{p}^{2}=.50$ for the second NRT session).

\section{*** Insert Table 3 and Figure 3 here $* * *$}

Finally, we used Fisher's Exact tests between subgroups to examine the type of knowledge transition from preNok or prelmK for the first session to postlmK or postExK for the second session; postNok subgroups were excluded from these analyses. For prelmK participants, the results showed a significant difference between YoungSleep and OldSleep participants $(p=.041)$, while no difference was revealed between the older subgroups $(p>$ .05). These findings indicated that the YoungSleep participants who had already gained implicit knowledge before sleep had more probability to gain explicit insight into the hidden rule (i.e. second response equals the final result) while older participants, irrespective of the type of consolidation interval, had a higher probability to only maintain implicit knowledge. A different pattern of results was obtained for the preNok participants who remained mostly in the similar knowledge level after the consolidation interval. No difference was found between the YoungSleep and the OldSleep groups $(p>.05)$, nor between older subgroups (Sleep vs Wakefulness; $p>.05$ ).

\section{DISCUSSION}

The present study aimed to investigate whether insight problem solving is promoted by sleep in aged participants like this was previously demonstrated in young adults. As 
expected, half of the young participants gained insight into the NRT hidden rule following a night of sleep. This result is in line with those obtained by Wagner and colleagues (2004) who have been the first to address whether sleeping on a problem can elicit the emergence of creative solutions. Comparing participants who either slept during the interval between the first and the second NRT sessions, or remained awake (either during daytime or night time), they found that participants who slept more than doubled the likelihood of solving the NRT using the hidden rule (59\%), compared to the other groups ( 20\% for each). In a similar vein, Cai et al. (2009) and Ritter et al. (2012) also reported a beneficial impact of sleep for creative insight in young adults. Although we did not include a young wake group in this study, our data showed that sleep benefits insight problem solving in young participants compared to older adults.

The major and innovative finding of the present study is that, after a night of sleep, the old adults did not gain as much insight as did the young adults. Furthermore, older adult groups gained insight with a comparable magnitude ( 20\%), irrespective of whether they had a night of sleep or a day of wakefulness. While age-related changes in sleep may have contributed to limit the beneficial effect of sleep, the present study was performed in old participants with a good quality of sleep, as evidenced by the absence of any significant difference in the total sleep time or in sleep efficiency between the young and the older groups. Yet, it is still possible that changes in the architecture of sleep or in some features of sleep, such as slow oscillatory activity, may have resulted in the observed pattern of results. So far, several investigations have reported that the most prominent changes in sleep architecture with aging is the reduction in the deepest stages of SWS (Cajochen, et al., 2006,Carrier, et al., 2001) along with increased lighter NREM sleep (stages N1 and N2), 
although the amount of REM sleep declines only slightly (Floyd, et al., 2007). As far as insight problem solving is concerned, Born and Wilhelm (2012) stated that SWS might mediate the restructuring of newly encoded memory representations by reactivating the connection between the prefrontal cortical areas and the hippocampus. Based on the findings mentioned above, we might speculate that the neural reactivation of NRT representations elicited between these regions might be insufficient to protract insight because of agerelated declines in SWS. This finding is consistent with a recent brain stimulation study that demonstrated, for the first time, the causal connection between SWS and the consolidation of declarative memory in older adults (Westerberg, et al., 2015). Nevertheless, the number of old participants who gained insight overnight did not exceed that of the corresponding wake group, which suggests an alternative or complementary explanation that insightrelated processes in older adults might be less efficient, possibly because of age-related brain metabolic, structural, and functional declines. Using NRT during wakefulness, Rose et al. (2010) demonstrated that the emergence of insight was mediated by the interaction between activation in prefrontal cortex and the medial temporal lobe. This finding is in line with the recent study of Milivojevic et al. (2015) that found such key role involvement of the prefrontal cortex and the hippocampus to solve a narrative-insight task. Paralleling the hypothesized reactivation of these same brain regions during sleep (Born and Wilhelm, (2012), these findings converge to suggest that the present results may more specifically reflect age related changes in the prefrontal cortex and hippocampus (Giovanello and Schacter, 2012,Resnick, et al., 2003,Tromp, et al., 2015,Yonelinas, et al., 2007), which would thus affect insight problem solving largely independently of sleep-related processes. Overall, more research in this field will need to disentangle whether changes in SWS or structural and 
functional neural alterations with aging are the primary cause of reduced overnight insight problem solving.

The present data further revealed that acquisition of implicit knowledge before sleep potentiated the emergence of insight the day after in the young participants, but not in the old participants. Interestingly, the latter rather conserved their implicit level of knowledge both after sleep and wakefulness periods. Our results in young participants is reminiscent of the finding of Yordonova et al. (2008) who showed that young participants who acquired implicit knowledge before sleep, compared to those who did not, increase the opportunity to gain insight of the NRT regularity the day after. Interestingly, they further demonstrated a selective effect of early-night sleep (i.e. rich in SWS) on this higher gain of insight, while latenight sleep (i.e. rich in REM) rather allowed for the stabilization of implicit knowledge during the second NRT session. In addition, Darseau et al. (2011) showed that it is possible to distinguish between participants who will gain insight overnight and those who won't, based on their neural responses during the first pre-sleep NRT session. Our results showed no age differences in the acquisition of implicit knowledge during the first NRT session, older adults mostly maintained this level of knowledge on the task, while younger participants switched comparatively more often from implicit to explicit knowledge (or insight). Several authors have reported that aging is accompanied with declines in explicit forms of learning but not in implicit aspects of learning (e.g. the serial reaction time task; Bo and Seidler, 2010,Verwey, et al., 2011). For example, using an alternating serial reaction time task (ASRTT), Verneau et al. (2014) reported that implicit learning was preserved in old as much as in young adults, while explicit learning declined with age, the latter likely relating to the time constraints of the task that may hinder explicit learning. Therefore, it is reasonable to assume that the time 
pressure intrinsic to the NRT paradigm might have limited the possibility to gain explicit knowledge in the older groups. Alternatively, we might consider the young participants who were SWS-deprived in Yordonova's study (2008) as an experimental model for age-related SWS decline. When comparing the data in Yordonova's SWS-deprived group and our old sleep group, it is interesting to observe that both remained at the implicit level of performance during the second NRT session. Taken together, these findings support the hypothesis that SWS may indeed facilitate the emergence of insight when implicit knowledge was acquired before sleep, while REM sleep may stabilize recently acquired implicit knowledge. Hence, future investigation using polysomnographic assessment should help to further establish the role sleep in the transformation of implicit intuitions into the suitable and explicit resolution of complex problems in older adults.

In summary, our results provide evidence that sleep does not potentiate creative problem solving in older adults as it does in young adults. We also found that although older adults can acquire implicit knowledge about problem solving to a same extent as younger participants, implicit knowledge in old adults does not subsequently promote explicit knowledge or insight (after sleep or after wakefulness). These findings underline distinct factors that may underlie reduced overnight problem solving in older adults. It is likely that decline in SWS might have a direct influence on age-related consolidation and especially on the insight problem solving. Likewise, impairment in hippocampal-neocortical functional connectivity might have impacted memory consolidation process, independently of the SWS (Scullin and Bliwise, 2015). The present findings may therefore stimulate further investigations on the impact of sleep on age-related neurobiological changes affecting memory functions. 


\section{References}

Backhaus, J., Born, J., Hoeckesfeld, R., Fokuhl, S., Hohagen, F., Junghanns, K. 2007. Midlife decline in declarative memory consolidation is correlated with a decline in slow wave sleep. Learn Mem 14(5), 336-41. doi:10.1101/Im.470507.

Beck, A.T., Ward, C.H., Mendelson, M., Mock, J., Erbaugh, J. 1961. An inventory for measuring depression. Arch Gen Psychiatry 4, 561-71.

Bo, J., Seidler, R.D. 2010. Spatial and symbolic implicit sequence learning in young and older adults. Exp Brain Res 201(4), 837-51. doi:10.1007/s00221-009-2098-5.

Born, J., Wilhelm, I. 2012. System consolidation of memory during sleep. Psychol Res 76(2), 192-203. doi:10.1007/s00426-011-0335-6.

Buhry, L., Azizi, A.H., Cheng, S. 2011. Reactivation, replay, and preplay: how it might all fit together. Neural Plast 2011, 203462. doi:10.1155/2011/203462.

Buysse, D.J., Reynolds, C.F., 3rd, Monk, T.H., Berman, S.R., Kupfer, D.J. 1989. The Pittsburgh Sleep Quality Index: a new instrument for psychiatric practice and research. Psychiatry Res 28(2), 193-213. doi:0165-1781(89)90047-4 [pii].

Cai, D.J., Mednick, S.A., Harrison, E.M., Kanady, J.C., Mednick, S.C. 2009. REM, not incubation, improves creativity by priming associative networks. Proc Natl Acad Sci U S A 106(25), 10130-4. doi:10.1073/pnas.0900271106.

Cajochen, C., Munch, M., Knoblauch, V., Blatter, K., Wirz-Justice, A., Carrier, J., Land, S., Buysse, D.J., Kupfer, D.J., Monk, T.H. 2006. Age-related changes in the circadian and homeostatic regulation of human sleep. Chronobiol Int 23(1-2), 461-74. 
Carcaillon, L., Amieva, H., Auriacombe, S., Helmer, C., Dartigues, J.F. (2009). A subtest of the MMSE as a valid test of episodic memory? Comparison with the Free and Cued Reminding Test. Dement Geriatr Cogn Disord 27(5), 429-38. doi: 10.1159/000214632.

Carrier, J., Land, S., Buysse, D.J., Kupfer, D.J., Monk, T.H. 2001. The effects of age and gender on sleep EEG power spectral density in the middle years of life (ages 20-60 years old). Psychophysiology 38(2), 232-42.

Carskadon, M., Rechtschaffen, A. 2005. Monitoring and staging human sleep. in: Kryger, M., Roth, T., Dement, W. (Eds.). Principles and Practice of Sleep Medicine. Elsevier Saunders, Philadelphia:, pp 1359-77.

Darsaud, A., Wagner, U., Balteau, E., Desseilles, M., Sterpenich, V., Vandewalle, G., Albouy, G., Dang-Vu, T., Collette, F., Boly, M., Schabus, M., Degueldre, C., Luxen, A., Maquet, P. 2011. Neural precursors of delayed insight. J Cogn Neurosci. 23(8), 1900-10. doi: 10.1162/jocn.2010.21550.

Diekelmann, S., Born, J. 2010. The memory function of sleep. Nature reviews Neuroscience 11(2), 114-26. doi:10.1038/nrn2762.

Doyon, J., Korman, M., Morin, A., Dostie, V., Hadj Tahar, A., Benali, H., Karni, A., Ungerleider, L.G., Carrier, J. 2009. Contribution of night and day sleep vs. simple passage of time to the consolidation of motor sequence and visuomotor adaptation learning. Exp Brain Res 195(1), 15-26. doi:10.1007/s00221-009-1748-y [doi].

Floyd, J.A., Janisse, J.J., Jenuwine, E.S., Ager, J.W. 2007. Changes in REM-sleep percentage over the adult lifespan. Sleep 30(7), 829-36.

Folstein, M.F., Folstein, S.E., McHugh, P.R. 1975. "Mini-mental state". A practical method for grading the cognitive state of patients for the clinician. J Psychiatr Res 12(3), 189-98. 
Giovanello, K.S., Schacter, D.L. 2012. Reduced specificity of hippocampal and posterior ventrolateral prefrontal activity during relational retrieval in normal aging. J Cogn Neurosci 24(1), 159-70. doi:10.1162/jocn_a_00113.

Haider, H., Rose, M. 2007. How to investigate insight: a proposal. Methods 42(1), 49-57. doi:10.1016/j.ymeth.2006.12.004.

Harand, C., Bertran, F., Doidy, F., Guenole, F., Desgranges, B., Eustache, F., Rauchs, G. 2012. How aging affects sleep-dependent memory consolidation? Front Neurol 3, 8 . doi:10.3389/fneur.2012.00008.

Hoddes, E., Dement, W.C., Zarcone, V. 1972. The development and use of the Stanford sleepiness scale. Psychophysiology 9, 150.

Igloi, K., Gaggioni, G., Sterpenich, V., Schwartz, S. 2015. A nap to recap or how reward regulates hippocampal-prefrontal memory networks during daytime sleep in humans. elife 4. doi:10.7554/eLife.07903.

Klerman, E.B., Wang, W., Duffy, J.F., Dijk, D.J., Czeisler, C.A., Kronauer, R.E. 2013. Survival analysis indicates that age-related decline in sleep continuity occurs exclusively during NREM sleep. Neurobiol Aging 34(1), 309-18. doi: 10.1016

Mander, B.A., Rao, V., Lu, B., Saletin, J.M., Ancoli-Israel, S., Jagust, W.J., Walker, M.P. 2014. Impaired prefrontal sleep spindle regulation of hippocampal-dependent learning in older adults. Cerebral cortex (New York, NY : 1991) 24(12), 3301-9. doi:10.1093/cercor/bht188.

Milivojevic, B., Vicente-Grabovetsky, A., Doeller, C.F. 2015. Insight reconfigures hippocampal-prefrontal memories. Curr Biol 25(7), 821-30. doi:10.1016/j.cub.2015.01.033. Milner, B. 1971. Interhemispheric differences in the localization of psychological processes in man. Br Med Bull 27(3), 272-7. 
Nemeth, D., Janacsek, K., Londe, Z., Ullman, M.T., Howard, D.V., Howard, J.H., Jr. 2010. Sleep has no critical role in implicit motor sequence learning in young and old adults. Exp Brain Res 201(2), 351-8. doi:10.1007/s00221-009-2024-x.

Petit, D., Gagnon, J.F., Fantini, M.L., Ferini-Strambi, L., Montplaisir, J. 2004. Sleep and quantitative EEG in neurodegenerative disorders. J Psychosom Res 56(5), 487-96. doi:10.1016/j.jpsychores.2004.02.001.

Reitan, R.M. 1992. Trail Making Test: Manual for administration and scoring, Tucson

Resnick, S.M., Pham, D.L., Kraut, M.A., Zonderman, A.B., Davatzikos, C. 2003. Longitudinal magnetic resonance imaging studies of older adults: a shrinking brain. The Journal of neuroscience : the official journal of the Society for Neuroscience 23(8), 3295-301.

Ritter, S.M., Strick, M., Bos, M.W., van Baaren, R.B., Dijksterhuis, A. 2012. Good morning creativity: task reactivation during sleep enhances beneficial effect of sleep on creative performance. J Sleep Res 21(6), 643-7. doi:10.1111/j.1365-2869.2012.01006.x.

Robertson, E.M. 2009. From creation to consolidation: a novel framework for memory processing. PLoS Biol 7(1), e19. doi:10.1371/journal.pbio.1000019.

Rose, M., Haider, H., Büchel, C. 2010. The emergence of explicit memory during learning. Cereb Cortex 20(12), 2787-97. doi:10.1093/cercor/bhq025.

Scullin, M.K. 2012. Sleep, Memory, and Aging: The Link Between Slow-Wave Sleep and Episodic Memory Changes From Younger to Older Adults. Psychol Aging. doi:10.1037/a0028830.

Scullin, M.K., Bliwise, D.L. 2015. Sleep, cognition, and normal aging: integrating a half century of multidisciplinary research. Perspect Psychol Sci 10(1), 97-137. doi:10.1177/1745691614556680. 
Song, S. 2009. Consciousness and the consolidation of motor learning. Behav Brain Res 196(2), 180-6. doi:S0166-4328(08)00549-4 [pii] 10.1016/j.bbr.2008.09.034 [doi].

Spencer, R.M., Sunm, M., Ivry, R.B. 2006. Sleep-dependent consolidation of contextual learning. Curr Biol 16(10), 1001-5. doi:S0960-9822(06)01560-0 [pii] 10.1016/j.cub.2006.03.094 [doi].

Stickgold, R., Walker, M.P. 2007. Sleep-dependent memory consolidation and reconsolidation. Sleep Med 8(4), 331-43. doi:S1389-9457(07)00077-9 [pii] 10.1016/j.sleep.2007.03.011 [doi].

Tamminen, J., Payne, J.D., Stickgold, R., Wamsley, E.J., Gaskell, M.G. 2010. Sleep spindle activity is associated with the integration of new memories and existing knowledge. The Journal of neuroscience : the official journal of the Society for Neuroscience 30(43), 1435660. doi:10.1523/jneurosci.3028-10.2010.

Thurstone, L.L., Thurstone, T.G. 1941. Factorial studies of intelligence. Psychometr Monogr 2,94 .

Tromp, D., Dufour, A., Lithfous, S., Pebayle, T., Despres, O. 2015. Episodic memory in normal aging and Alzheimer disease: Insights from imaging and behavioral studies. Ageing Res Rev. doi:10.1016/j.arr.2015.08.006.

Tucker, M., McKinley, S., Stickgold, R. 2011. Sleep optimizes motor skill in older adults. J Am Geriatr Soc 59(4), 603-9. doi:10.1111/j.1532-5415.2011.03324.x.

Van Cauter, E., Leproult, R., Plat, L. 2000. Age-related changes in slow wave sleep and REM sleep and relationship with growth hormone and cortisol levels in healthy men. JAMA 284(7), 861-8. 
Van Der Werf, Y.D., Altena, E., Schoonheim, M.M., Sanz-Arigita, E.J., Vis, J.C., De Rijke, W., Van Someren, E.J. 2009. Sleep benefits subsequent hippocampal functioning. Nature neuroscience 12(2), 122-3. doi:10.1038/nn.2253.

Verneau, M., van der Kamp, J., Savelsbergh, G.J., de Looze, M.P. 2014. Age and time effects on implicit and explicit learning. Exp Aging Res 40(4), 477-511. doi:10.1080/0361073x.2014.926778.

Verwey, W.B., Abrahamse, E.L., Ruitenberg, M.F., Jimenez, L., de Kleine, E. 2011. Motor skill learning in the middle-aged: limited development of motor chunks and explicit sequence knowledge. Psychol Res 75(5), 406-22. doi:10.1007/s00426-011-0320-0.

Wagner, U., Gais, S., Haider, H., Verleger, R., Born, J. 2004. Sleep inspires insight. Nature 427(6972), 352-5. doi:10.1038/nature02223.

Walker, M.P., Stickgold, R. 2010. Overnight alchemy: sleep-dependent memory evolution. Nature reviews Neuroscience 11(3), 218; author reply doi:nrn2762-c1 [pii] 10.1038/nrn2762c1 [doi].

Wechsler, D. 1981. The Wechsler Adult Intelligence Scale. The Psychological Corporation., New York.

Westerberg, C.E., Florczak, S.M., Weintraub, S., Mesulam, M.M., Marshall, L., Zee, P.C., Paller, K.A. 2015. Memory improvement via slow-oscillatory stimulation during sleep in older adults. Neurobiol Aging 36(9), 2577-86. doi:10.1016/j.neurobiolaging.2015.05.014.

Wilson, J.K., Baran, B., Pace-Schott, E.F., Ivry, R.B., Spencer, R.M. 2012. Sleep modulates word-pair learning but not motor sequence learning in healthy older adults. Neurobiol Aging 33(5), 991-1000. doi:10.1016/j.neurobiolaging.2011.06.029. 
Yesavage, J.A., Brink, T.L., Rose, T.L., Lum, O., Huang, V., Adey, M., Leirer, V.O. 1982. Development and validation of a geriatric depression screening scale: a preliminary report. J Psychiatr Res 17(1), 37-49.

Yonelinas, A.P., Widaman, K., Mungas, D., Reed, B., Weiner, M.W., Chui, H.C. 2007. Memory in the aging brain: doubly dissociating the contribution of the hippocampus and entorhinal cortex. Hippocampus 17(11), 1134-40. doi:10.1002/hipo.20341.

Yordanova, J., Kolev, V., Verleger, R. 2009. Awareness of knowledge or awareness of processing? Implications for sleep-related memory consolidation. Front Hum Neurosci 3, 40. doi:10.3389/neuro.09.040.2009.

Yordanova, J., Kolev, V., Verleger, R., Bataghva, Z., Born, J., Wagner, U. 2008. Shifting from implicit to explicit knowledge: different roles of early- and late-night sleep. Learn Mem 15(7), 508-15. doi:10.1101/Im.897908.

Acknowledgements: This work was supported by the National Center of Competence in Research (NCCR) Affective Sciences financed by the Swiss National Science Foundation ( $\mathrm{n}^{\circ}$ 51NF40-104897) and hosted by the University of Geneva, and by individual project grants from the Swiss National Science Foundation ( $n^{\circ} 320030-159862$ to S.S.) 


\section{Figure Captions}

Figure 1: Number Reduction Task (NRT). In the illustrated and typical example, the stimulus string was always composed by the digits ' 1 ', ' 4 ' and ' 9 '. The goal of the task is to determine the final digit result. To do so, subjects can perform sequential processing of digits pairwise from the left to the right according to two simple rules: the 'same rule', which states that the result of two identical digits is this same digit (response 1 here); the 'different rule', which states that the result of two non-identical digits is the remaining third digit of this three-digit system (responses 2 and 3 here). Thus, each response string is composed by seven response digits with the last being final result. Importantly, each pattern of response string contains a hidden rule such that the responses 2-4 always mirror the responses $5-7$, hence the second digit response is systematically the final result digit. Whenever participants gain explicit knowledge (insight) of this hidden rule, their response time to give the final result digit suddenly and significantly decreases.

Figure 2: Insight gains expressed in percent for the YoungSleep, OldSleep and OldWake groups. This shows that the YoungSleep group gained significantly more insight after a night of sleep than the OldSleep group, while no difference was found between both old groups (Sleep vs. Wakefulness).

Figure 3: Percentage of participants who did change or did not change their level of knowledge between the first and second NRT session. 
Figure 1

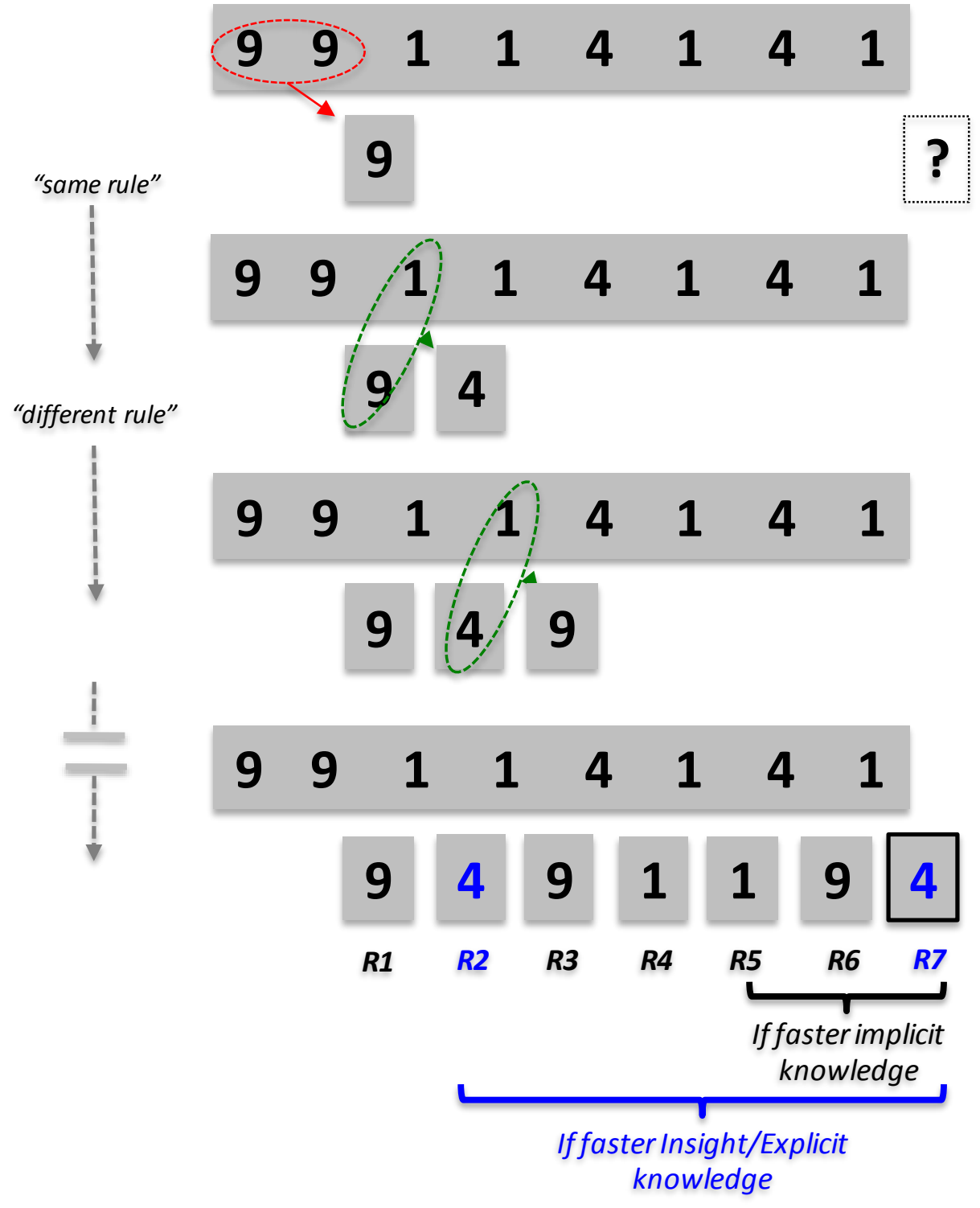


Figure 2

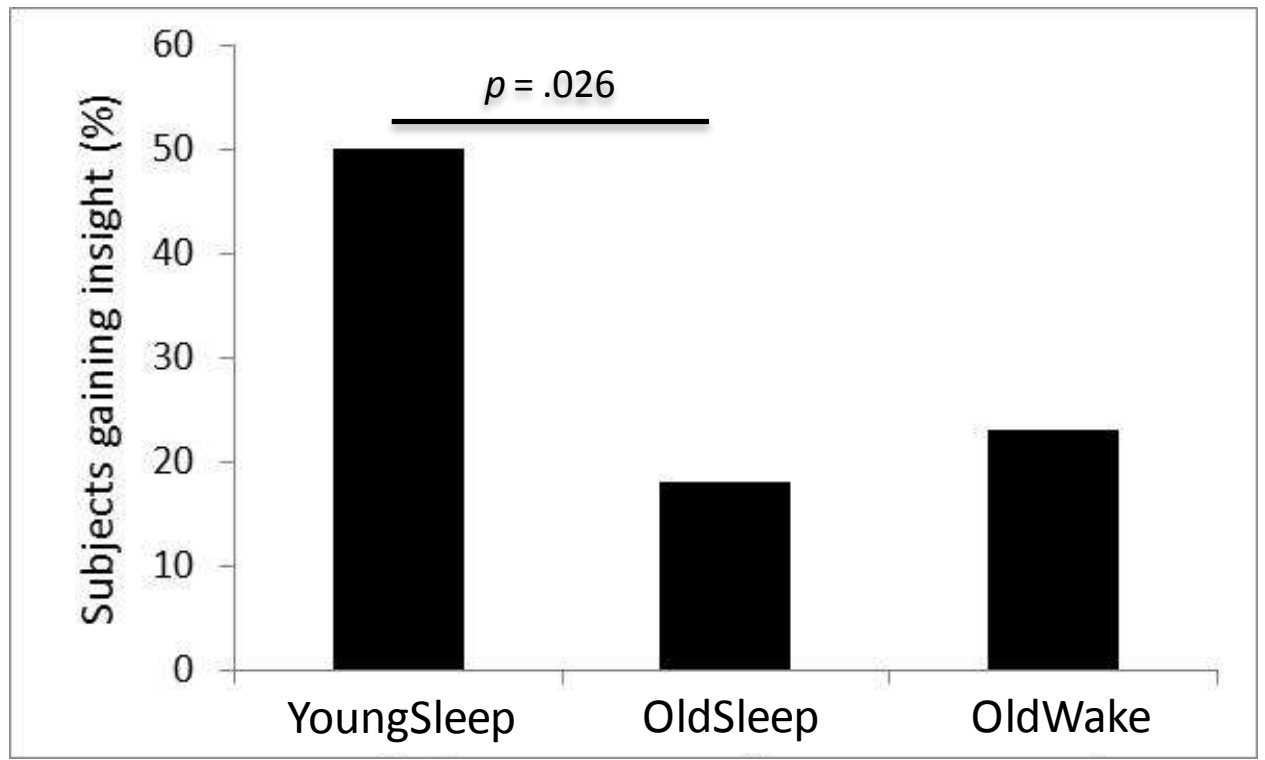


Figure 3

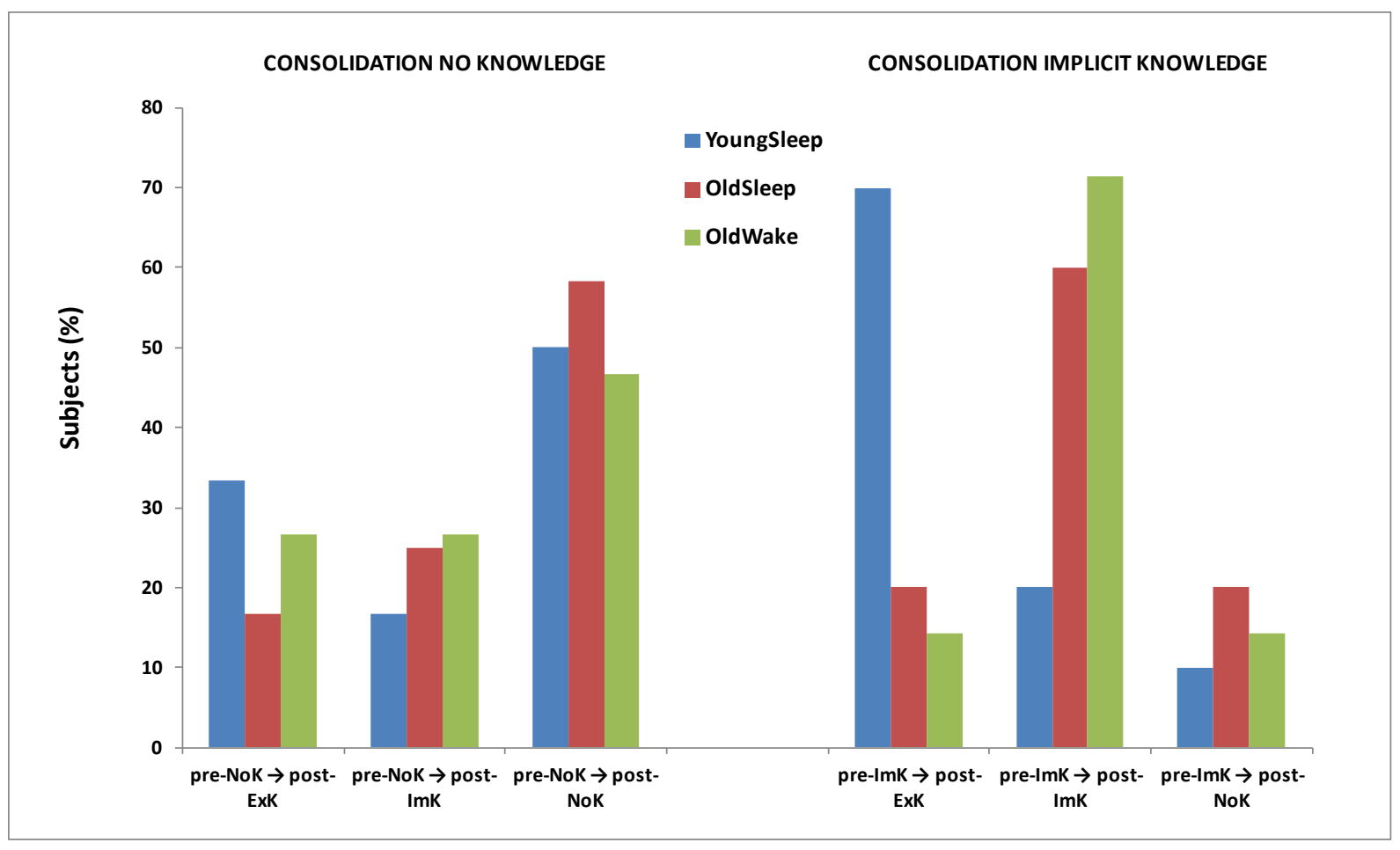


Table 1: Neuropsychological assessment (mean and SD) for the young and old groups. Age column refers to the difference between YoungSleep and Old groups (Sleep and Wake). Consolidation type column corresponds to the comparison between OldSleep and OldWake groups.

\begin{tabular}{|c|c|c|c|c|c|}
\hline (Mean, SD) & YoungSleep & OldSleep & OldWake & Age & $\begin{array}{c}\text { Consolidation } \\
\text { type }\end{array}$ \\
\hline \multicolumn{6}{|l|}{ Working memory } \\
\hline \multicolumn{6}{|l|}{ Digit Span } \\
\hline Forward & $7.68(.30)$ & $6.82(.30)$ & $6.38(.22)$ & $p=.02$ & $p=.28$ \\
\hline Backward & $6.91(.31)$ & $5.91(.36)$ & $6.14(.37)$ & $p=.35$ & $p=.71$ \\
\hline Corsi block span & $6.45(.19)$ & $5.05(.20)$ & $5.52(.22)$ & $p=.01$ & $p=.16$ \\
\hline \multicolumn{6}{|l|}{ Executive functions } \\
\hline Trail making test $A$ & $25.64(1.45)$ & $32.50(1.67)$ & $32.86(2.03)$ & $p=.01$ & $p=.65$ \\
\hline Trail making test $\mathrm{B}$ & $53.45(4.50)$ & $74.23(5.28)$ & $65.00(3.90)$ & $p=.01$ & $p=.23$ \\
\hline Trail making test $\mathrm{B}-\mathrm{A}$ & $27.82(3.86)$ & $41.73(4.69)$ & $32.14(1.87)$ & $p=.09$ & $p=.12$ \\
\hline \multicolumn{6}{|l|}{ Depression } \\
\hline \multicolumn{6}{|l|}{ assessment } \\
\hline \multicolumn{6}{|l|}{ inventory } \\
\hline Geriatric Depression & & $3.31(.55)$ & $3.47(.55)$ & & $p=.94$ \\
\hline \multicolumn{6}{|l|}{ Scale } \\
\hline MMSE & & $29,59(.11)$ & $29.71(.10)$ & & $p=.32$ \\
\hline
\end{tabular}


Table 2: Summary of the actigraphy data for the YoungSleep and OldSleep groups. Values for actigraphy are reported in hours/percentages as indicated.

\begin{tabular}{llcc}
\hline \multicolumn{1}{c}{ Sleep measures } & YoungSleep & OldSleep & $\boldsymbol{P}$ \\
\hline \multicolumn{1}{c}{ Actigraphy (hh:mm) } & & & \\
Bed time & $00: 42 \pm 01: 40$ & $11: 25 \pm 01: 05$ & $\mathrm{p}=.02$ \\
Sleep latency & $00: 11 \pm 00: 11$ & $00: 14 \pm 00: 10$ & $\mathrm{p}=.47$ \\
Sleep start & $00: 55 \pm 01: 44$ & $11: 39 \pm 01.09$ & $\mathrm{p}=.03$ \\
Sleep end & $08: 08 \pm 01: 16$ & $06: 37 \pm 01: 05$ & $\mathrm{P}=.01$ \\
Get up time & $08: 17 \pm 01: 27$ & $06: 51 \pm 01: 01$ & $\mathrm{P}=.01$ \\
Time Sleep Period & $07: 14 \pm 01: 35$ & $06: 57 \pm 01: 05$ & $\mathrm{p}=.58$ \\
Total Sleep Time & $07: 07 \pm 01: 31$ & $06: 50 \pm 01: 06$ & $\mathrm{p}=.59$ \\
Sleep efficiency (\%) & $93.89 \% \pm 0.90$ & $92.28 \% \pm 0.68$ & $\mathrm{p}=.25$ \\
\hline
\end{tabular}


Table 3: Assignment of participants with respect to knowledge acquired before and after consolidation interval. Subgroups are represented according to the number of participants and percentage of participants per group. PreNok and prelmK indicate no or implicit knowledge either before sleep of wakefulness interval; postNok, no knowledge during postsleep or wake intervals; postlmK, implicit knowledge during post-sleep or wake intervals, postExK, explicit knowledge or insight of the hidden rule during post-sleep or wake intervals; grey arrow, transition of knowledge state from pre- to post-sleep.

\begin{tabular}{|c|c|c|c|c|c|}
\hline & \multicolumn{5}{|c|}{ PRE-NoK } \\
\hline & TOTAL & Pre-NoK & Pre-NoK & Pre-NoK & Pre-NoK \\
\hline & & & Post-NoK & Post-ImK & Post-ExK \\
\hline YoungSleep & 22 & $12(54.5 \%)$ & $6(50 \%)$ & $2(16.7 \%)$ & $4(33.3 \%)$ \\
\hline OldSleep & 22 & $12(54.5 \%)$ & 7 (58.3) & $3(25 \%)$ & $2(16.7 \%)$ \\
\hline \multirow[t]{4}{*}{ OldWake } & 22 & 15 (68.2\%) & $7(46.6 \%)$ & $4(26.7 \%)$ & $4(26.7 \%)$ \\
\hline & \multicolumn{5}{|c|}{ PRE-ImK } \\
\hline & TOTAL & Pre-ImK & Pre-ImK & Pre-ImK & Pre-ImK \\
\hline & & & Post-NoK & Post-ImK & Post-ExK \\
\hline YoungSleep & 22 & $10(45.5 \%)$ & $1(10 \%)$ & $2(20 \%)$ & $7(70 \%)$ \\
\hline OldSleep & 22 & $10(45.5 \%)$ & $2(20 \%)$ & $6(60 \%)$ & $2(20 \%)$ \\
\hline OldWake & 22 & 7 (31.8\%) & $1(14.3 \%)$ & $5(71.4 \%)$ & 1 (14.3\%) \\
\hline
\end{tabular}


